IP Periodica Polytechnica Chemical Engineering

61(1), pp. 33-38, 2017

DOI: $10.3311 /$ PPch.9726

Creative Commons Attribution (i)

RESEARCH ARTICLE

\section{Relationship between Individual and Competitive Adsorption Isotherms on Molecularly Imprinted Polymers}

\author{
Zsanett Dorkó ${ }^{1,2}$, Barbara Tamás ${ }^{1}$, George Horvai ${ }^{1,2, *}$
}

Received 11 July 2016; accepted after revision 21 September 2016

\begin{abstract}
Molecularly imprinted polymers (MIP) are a new generation of selective adsorbents. In practical applications of MIPS simultaneous adsorption of at least two compounds occurs. Simultaneous (typically competitive) adsorption on MIPs has not yet been quantitatively analyzed. This paper shows that with a typical type of MIP the individual isotherms of two compounds coincide with their competitive isotherms in the $\log D$ $\log q$ isotherm plot, where $D$ is the distribution coefficient and $q$ is the adsorbed concentration. Based on this observation the usual competitive isotherm, i.e., the $\left(c_{1}, c_{2}\right)$ to $\left(q_{1}, q_{2}\right)$ mapping can be established from the two individual isotherms. (The $c-s$ are the respective solution phase equilibrium concentrations.) Batch separation experiments can be easily designed and the selectivity of the MIPs is also easily determined without the tedious measurement of the full competitive isotherm.
\end{abstract}

\section{Keywords}

molecularly imprinted polymer, adsorption isotherm, competitive adsorption isotherm, propranolol, beta blocker

\footnotetext{
${ }^{1}$ Department of Inorganic and Analytical Chemistry, Faculty of Chemical Technology and Biotechnology, Budapest University of Technology and Economics, H-1111 Budapest, Hungary

${ }^{2}$ MTA-BME Research Group of Technical Analytical Chemistry, H-1111 Budapest, Hungary

*Corresponding author, e-mail: george.horvai@mail.bme.hu
}

\section{Introduction}

Molecularly imprinted polymers (MIP) are a new generation of selective adsorbents. They are the subject of vigorous research which produces hundreds of papers annually. After years of fundamental research the time appears to be ripe now for their practical applications. This is attested by the increasing number of patent applications (62 in the year 2015 [1]). MIPs may be used as chromatographic stationary phases (e.g., for chiral separations) [2-5], as solid phase extraction (SPE) materials in analytical sample preparation [6, 7], in different variants of capillary electrophoresis [8], as sensor materials [9-11], as artificial antibodies [12], as catalysts [13, 14], as slow release vehicles of pharmaceuticals $[15,16]$, as adsorbents for selective removal of contaminants $[17,18]$ and as membrane materials [19].

MIPs are typically made by polymerization of suitable monomers in the presence of a so-called template compound. After polymerization the template is removed from the polymer. This procedure leaves empty binding sites in the polymer, which are chemical and geometrical imprints of the template molecule. Due to these sites the MIP can rebind from solutions the template or other molecules which are chemically related to the template. The rebinding on a good MIP occurs selectively against compounds which are not very closely related to the template. The target compound of a practical application can be either the template or a closely related compound (e.g. if the template is expensive or toxic or its bleeding would disturb). Eventually a group of closely related compounds to the template may be targeted.

For successful practical applications one needs to know if the MIP will show sufficient selectivity. Quantification of selectivity is a difficult problem in chemistry, and particularly in analytical chemistry [20-25]. In the case of MIPs one may rely on a large body of experience in relation to chromatographic adsorbents. The selectivity of liquid chromatographic stationary phases is easily characterized (in a given eluent) if the adsorption isotherms of all adsorbed solutes are linear. In this case selectivity between two adsorbable compounds can be given by the ratio of the respective isotherm slopes, i.e., by the ratio of the respective, 
concentration independent distribution coefficients. These distribution coefficients are the same in separate solutions of the respective compounds and in their mixtures, i.e., the phase distribution of the two compounds is mutually independent.

If at least one isotherm is not linear, the issue of selectivity becomes complex. Distribution coefficients are then concentration dependent, and the distribution of two compounds is usually not independent from each other. What one can do is to measure experimentally the isotherms of mixtures. In the case of only two compounds this means that different solution mixtures are equilibrated with the adsorbent and the resulting four equilibrium concentrations (one for each compound in each phase) are measured. These data quadruplets constitute the isotherm. More precisely the isotherm is a vector-vector function between the $\left(c_{1}, c_{2}\right)$ and the $\left(q_{1}, q_{2}\right)$ vectors, where the $c$-s are the solution concentrations of the two compounds and the $q$-s are their adsorbed concentrations. Often, but not always, the adsorption of both compounds is decreased by the presence of the other compound and therefore such four dimensional isotherms are called competitive isotherms. One should mention, however, that cooperative or synergistic adsorption is also possible. For a MIP this has been shown, e.g., by Pap [26].

Much knowledge has been collected about competitive isotherms of liquid chromatographic stationary phases [27]. Measuring the competitive isotherm in the general case requires a rather large number of experiments. The situation is better if the competitive isotherm can be constructed from the individual isotherms of the two compounds. Measurement of the individual isotherms requires much fewer and also more simple measurements than measuring the full four dimensional competitive isotherm.

Many papers on MIPs present the isotherm of the template and sometimes the individual isotherms of other compounds are also measured. Competitive isotherms of MIPs are, however, rarely studied. Several authors have reported competitive binding experiments [28-32] which were limited to the measurement of a few points, typically at a fixed concentration ratio of the two compounds. Apparently no relationship has been described between the individual and the competitive isotherms of MIPs.

In the present work individual and competitive isotherms of a widely studied MIP, the propranolol MIP [33-39] are studied. A simple relationship between the individual isotherms and the competitive isotherm is found. This relationship allows the design of practical MIP applications by using simple graphical procedures.

\section{Experimental}

\subsection{Materials}

Methacrylic acid (MAA), ethylene glycol dimethacrylate (EDMA), propranolol hydrochloride, dibenzylamine and $(R)$-(-)-2-benzylamino-1-phenylethanol were purchased from Sigma-Aldrich. Azobisisobutyronitrile (AIBN) was ordered from Fluka. $\mathrm{HCl}$ was obtained from Riedel-De Haën.
Acetonitrile, methanol and methyl tert-butyl ether were ordered from Merck. Water was purified with a Milli Q Direct 8 system.

\subsection{Instrumentation}

The following instruments were used: Grant-Bio PTR-35 multi-rotator, Eppendorf Minispin centrifuge, Perkin Elmer Series 200 HPLC (with UV detector), Purospher RP18-e $(125 \times 3 \mathrm{~mm}, 5 \mu \mathrm{m}$, Merck) reversed phase column.

\subsection{Synthesis of propranolol MIP}

Methacrylic acid (MAA) and ethylene glycol dimethacrylate (EDMA) were purified before use by using an inhibitor remover column (Sigma-Aldrich). Prior to use, propranolol hydrochloride was transformed to its free base form, by means of neutralization with $0.2 \mathrm{M} \mathrm{NaOH}$ solution followed by extraction with methyl tert-butyl ether.

Propranolol MIP was prepared as described previously, using the method of Andersson [33]. The polymerization mixture containing the template $(0.25 \mathrm{mmol})$, functional monomer (MAA, $2 \mathrm{mmol}$ ), crosslinker (EDMA, $10 \mathrm{mmol}$ ), initiator (AIBN, $0.15 \mathrm{mmol}$ ) and the polymerization solvent (ACN, $2.73 \mathrm{~mL}$ ) was prepared in a glass vial prior to polymerization. The mixture was purged with argon for 5 minutes, tightly sealed with a PTFE septum cap and was placed under an UV source (366 nm) for 24 hours. The formed bulk polymer was crushed. The polymer was washed several times with $0.01 \mathrm{M} \mathrm{HCl}$ solution in methanol-water 1:1, and then with methanol. After washing, the polymer was dried at room temperature overnight.

\subsection{Equilibrium binding experiments}

Equilibrium binding measurements were carried out at room temperature (ca. $26^{\circ} \mathrm{C}$ ). The temperature dependence of binding was checked to be negligible within $\pm 3^{\circ} \mathrm{C}$. Polymers were weighed in air dry state into polypropylene microtubes and the solution of analyte was pipetted into the tube. The adsorption isotherms were measured by applying different initial concentrations and volumes of the analyte solutions. During competitive isotherm measurement, propranolol and dibenzylamine were mixed in different ratios in $\mathrm{ACN}$. The concentration ratio of propranolol/dibenzylamine was changed from 0.5 to 2.5 .

After $30 \mathrm{~min}$ equilibration with mixing, the samples were centrifuged. This time was checked to be enough to reach the equilibrium. The supernatant was diluted with the HPLC eluent and injected into the HPLC system to quantify the unbound analyte concentration.

The chromatographic measurement of the analytes was accomplished on a Purospher RP18-e $(125 \times 3 \mathrm{~mm}, 5 \mu \mathrm{m}$, Merck) reversed phase column. The eluent flow rate was $0.6 \mathrm{~mL} / \mathrm{min}$, injection volume was $10 \mu \mathrm{L}$. The detection wavelength was set at $215 \mathrm{~nm}$. The eluent for propranolol measurement was phosphate buffer $\left(10 \mathrm{mM} \mathrm{NaH} \mathrm{PO}_{4}\right.$, the $\mathrm{pH}$ was adjusted to 3 by $\mathrm{H}_{3} \mathrm{PO}_{4}$ )-ACN 70:30 (retention time: $3.1 \mathrm{~min}$ ), and the eluent for 
dibenzylamine measurement was phosphate buffer (as above): ACN 75:25 (retention time: $1.7 \mathrm{~min}$ ).

\section{Results and discussion}

In this paper the binding isotherms of a well-researched MIP, the propranolol MIP are investigated. Propranolol is a widely used beta blocker drug. The MIP has been made with methacrylic acid (MAA) functional monomer and ethyleneglycol dimethacrylate (EDMA) crosslinker in acetonitrile (ACN) porogen.

\subsection{Experimental relationship between the individual and the competitive isotherms}

Figure 1 shows the isotherms of the template propranolol and of two other compounds. Both compounds are secondary amines, like propranolol. Dibenzylamine was chosen because it is a cheap compound, while $(R)$-(-)-2-benzylamino-1phenylethanol contains an alcoholic - $\mathrm{OH}$ group in beta position to the amino group, like propranolol. The isotherms have been measured by batch (static) adsorption method. The isotherms are plotted on a log-log scale, i.e, the log of the equilibrium adsorbed concentration, $q$ (in $\mathrm{mol} / \mathrm{kg}$ ), is plotted as a function of the log of the equilibrium solution concentration, $c$ (in $\mathrm{mol} / \mathrm{L}$ ). The reproducibility of the concentration measurements was about $2.5 \%$.

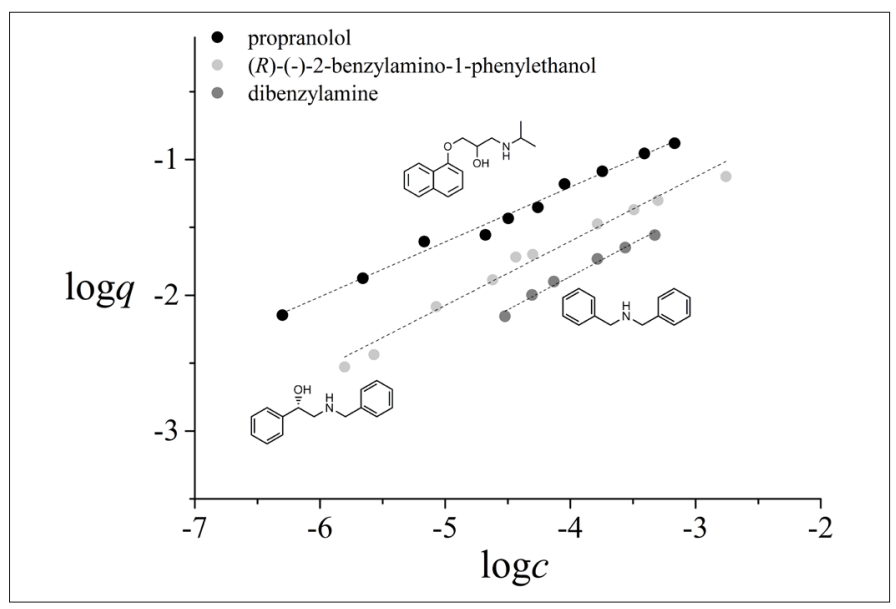

Fig. 1 Isotherms of secondary amines on propranolol MIP in the porogenic solvent $(\mathrm{ACN})$

As seen in Fig. 1 the isotherms are approximately linear in the $\log q-\log c$ plot in the studied concentration range, and the slopes of the fitted straight isotherm lines of different compounds are close to each other.

Figure 2 shows the individual isotherms of propranolol and dibenzylamine in a different form. Here the log of the distribution coefficient $D$ is plotted against $\log q$. The distribution coefficient is $D_{\mathrm{i}}=q_{\mathrm{i}} / c_{\mathrm{i}}$ where the index $\mathrm{i}$ denotes the individual compounds. This plot is indeed an adsorption isotherm because it can be easily obtained from the $q_{i}-c_{i}\left(\right.$ or $\left.\log q_{i}-\log c_{i}\right)$ isotherm and vice versa. The individual isotherms of the two compounds are shown by the circle symbols.

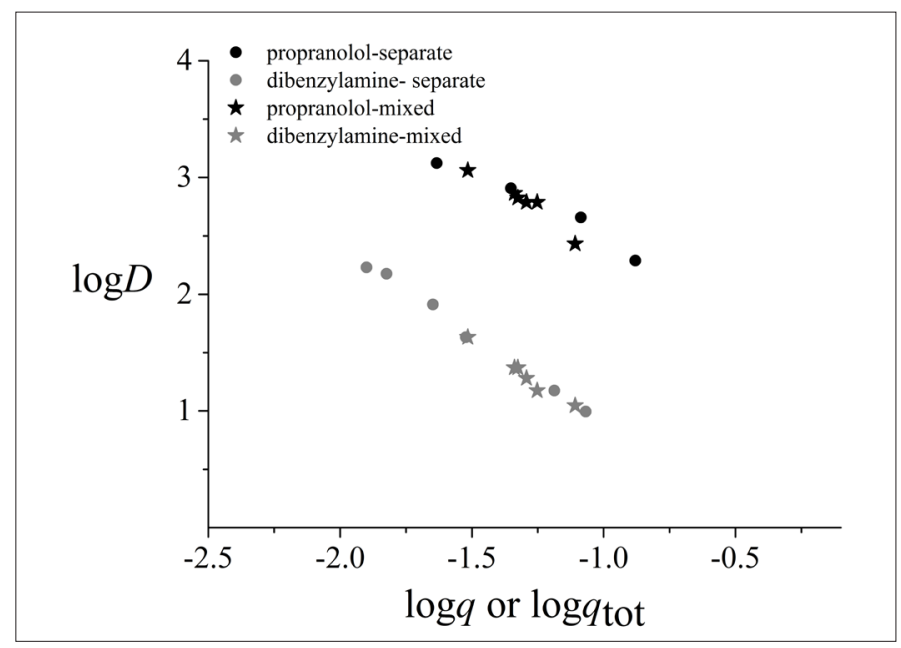

Fig. 2 Propranolol and dibenzylamine adsorption on propranolol MIP from separate and mixed solutions, respectively

Figure 2 includes also measurement points obtained in mixtures of propranolol and dibenzylamine. These points are denoted by asterisk symbols. For these mixed solutions the vertical axis shows again the distribution coefficients of the respective compounds. But on the horizontal axis the sum of the adsorbed concentrations of the two compounds is shown. In other words, the horizontal axis shows in this case the total adsorbed concentration (in $\mathrm{mol} / \mathrm{kg}$ ).

It strucks the eye immediately that in this special plotting method of Fig. 2 the individual and the competitive isotherms of either compound are practically overlapping. This means that a special form of the competitive isotherm, the $D_{\mathrm{i}}-q_{\text {tot }}$ isotherm is very simply defined by the individual isotherms.

In the next section it will be shown that, by using the just described feature of the $\log D-\log q$ plots, any four dimensional point of the competitive isotherm of these two compounds may be calculated from the two individual isotherms. The subsequent section will prove that one can also predict from the two individual isotherms the result of any batch separation of these two compounds with the MIP. Optimization of the separation can also be done without further experiments in mixtures.

\subsection{Calculation of the competitive isotherm from the individual isotherms}

One can calculate the full four dimensiomal competitive isotherm with the help of the empirical relationship found above between the individual isotherms and the competitive isotherm, respectively. This can be done by the following algorithm (as shown in Fig. 3).

1. Measure the individual isotherms and plot them as $\log D_{i}$ against $\log q_{i}$ as in Fig. 2 .

2. Take an arbitrary value of $q$ as $q_{\text {tot }}$ and determine the corresponding individual $D_{1}$ and $D_{2}$ values from the $\log D-\log q$ plot for compounds 1 and 2 , respectively.

3. Take any value of $q_{1}<q_{\mathrm{tot}}$. Then $q_{2}$ is given by $q_{\mathrm{tot}}-q_{1}$. From the two individual $D$ values the solution concentrations 
$c_{1}$ and $c_{2}$, respectively, can be calculated with the help of $q_{1}$ and $q_{2}$ as $c_{1}=q_{1} / D_{1}$ and $c_{2}=q_{2} / D_{2}$, respectively. The obtained values of $q_{1}$ and $q_{2}$ together with $c_{1}$ and $c_{2}$ satisfy the competitive isotherm.

4. Repeat the calculation in point 3 with some other $q_{1}$ values.

5. Redo the whole calculation of points 3 and 4 with some new $q_{\text {tot }}$ values.

\section{Fig. 2}

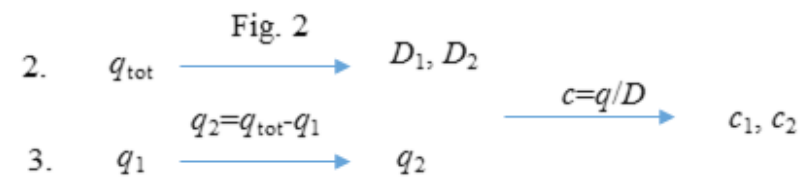

4. Repeat Step 3, with new $q_{1}$ value

5. Repeat Step 2, with new $q_{\text {tot }}$ value

\section{whole $\left(\mathrm{c}_{1}, \mathrm{c}_{2}, \mathrm{q}_{1}, \mathrm{q}_{2}\right)$ isotherm}

Fig. 3 Obtaining the whole competitive isotherm based on Fig. 2

This process produces any required number of (four dimensional) points of the competitive isotherm. Therefore the competitive isotherm can be calculated from the individual isotherms.

The approximate linearity of the isotherms in the $\log D-\log q$ plot has not been used in the above procedure. Thus the method is also valid if the isotherms are not linear in the $\log D-\log q$ plot. On the other hand, if they are linear, then a numerical calculation can replace the graphical one.

\subsection{Design of batch separations of two compounds with the MIP}

With the help of Fig. 2 one can design for example the (partial) separation of two compounds by a single batch adsorption on the MIP.

Let us assume that the raw sample contains $n_{1}$ and $n_{2}$ moles of the respective compounds 1 and 2 in a volume $V$. Extract this solution with MIP of mass $m$. Then:

$$
\begin{gathered}
q_{\mathrm{i}}=\left(n_{\mathrm{i}}-c_{\mathrm{i}} V\right) / m . \\
D_{\mathrm{i}}=\left(n_{\mathrm{i}}-c_{\mathrm{i}} V\right) / c_{\mathrm{i}} m . \\
q_{\text {tot }}=\left(\left(n_{1}+n_{2}\right)-\left(c_{1}+c_{2}\right) V\right) / m .
\end{gathered}
$$

The following algorithm allows the calculation of the result of this experiment, i.e., the equilibrium values of $c_{1}, c_{2}, q_{1}$ and $q_{2}$.

1. Using Eq. (3) one can make a linear plot of the mass balance in a coordinate system of $q_{\text {tot }}$ against $c_{\text {tot }}=c_{1}+c_{2}$. (Fig. 4)

2. On the other hand one can determine from Fig. 2 for any $q_{\text {tot }}$ value the corresponding $D_{1}$ and $D_{2}$ values. These allow the calculation of the values of $c_{1}$ and $c_{2}$ from Eq. (2) written for the respective $D$-s. Hence $c_{1}+c_{2}=c_{\text {tot }}$ can also be calculated. Since this $c_{\text {tot }}$ is the total solution concentration in equilibrium with the particular value of $q_{\text {tot }}$ chosen, one obtains an equilibrium point in the $q_{\text {tot }}-c_{\text {tot }}$ diagram.

3. Repeating the process described in point 2 for several $q_{\text {tot }}$ values, a curve of $q_{\text {tot }}$ vs $c_{\text {tot }}$ is obtained (Fig. 4). The intersection of this curve with the mass balance straight line gives the "working point". This working point shows the $c_{\text {tot }}$ and $q_{\text {tot }}$ values, respectively, which prevail at equilibrium in the experiment studied.

4. Having obtained the right $q_{\text {tot }}$ value for the experiment, one can find the corresponding $D_{1}$ and $D_{2}$ values in the $\log D-\log q_{\text {tot }}$ plot. From these $D_{i}$ values $c_{1}$ and $c_{2}$ can be calculated using Eq. (2). $q_{1}$ and $q_{2}$ are then calculated as $q_{1}=D_{1} c_{1}$ and $q_{2}=D_{2} c_{2}$.

Thus one obtains all equilibrium concentrations in the system, i.e., one can predict the result of a batch separation with the MIP from the two individual isotherms.

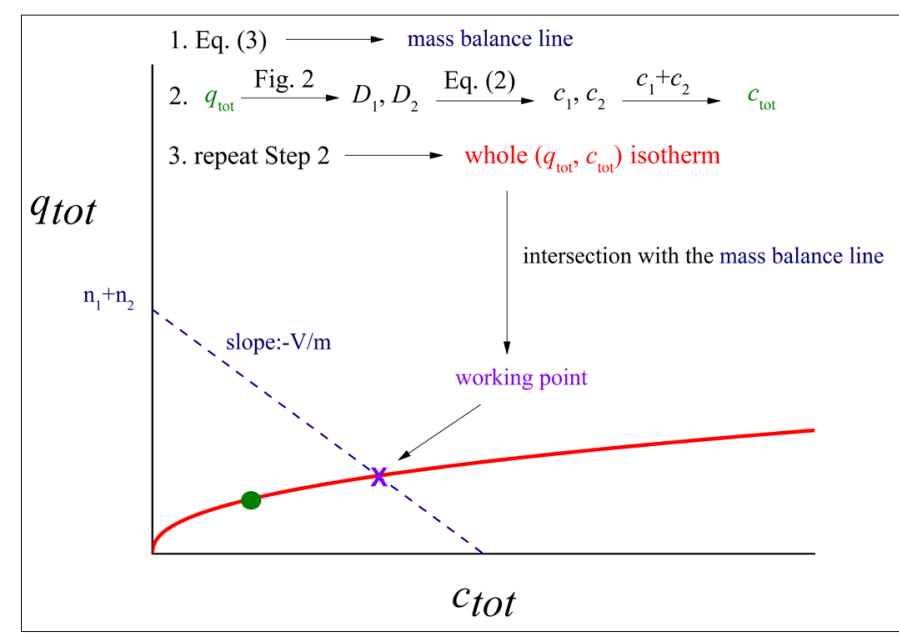

Fig. 4 Prediction of the result of a batch separation

Further on one can also easily predict the effect of changing the phase ratio $\mathrm{V} / \mathrm{m}$ or the starting concentrations $n_{i} / V$. In other words one can predict the outcome of any other design of the batch extraction.

Here again, if the linearity of the $\log D-\log q_{\text {tot }}$ isotherms is used, the calculations can be done without graphical manipulations. 


\subsection{Selectivity in mixed solutions}

Adsorption selectivity (or rather the adsorption selectivity factor) may be defined as the ratio of the respective distribution coefficients of the two compounds in a given extraction experiment: $\operatorname{Sel}=D_{1} / D_{2}$ or $\log S e l=\log \left(D_{1} / D_{2}\right)$. If one wishes to know the selectivity in a particular experiment, one has to carry out the same calculations as in Section 3.3 up to step 4. In step 4 the respective $D_{i}$ values of the two compounds are found for the particular experiment. Their ratio is the selectivity factor. The logarithm of the selectivity factor is the vertical distance in Fig. 2 between $\log D_{1}$ and $\log D_{2}$ at the $q_{\text {tot }}$ of the working point.

If the two $\log D-\log q_{\text {tot }}$ isotherms happen to be parallel in a plot like Fig. 2, then the selectivity is constant, i.e., it is the same in any experiment. Obviously the statement is strictly true only in the concentration range where the isotherms have been measured.

\subsection{Generalization of the method}

In the previous sections it has been shown that the competitive isotherm of the propranolol MIP with the compounds propranolol and dibenzylamine may be constructed from the respective individual isotherms. This method was based on the empirical observation that the competitive isotherms coincided with the respective individual isotherms in the $\log D-\log q$ plot. The question is now how far this result can be generalized to other MIPs and to other compound pairs. The example shown in Fig. 2 is special in that the individual isotherms are approximately straight lines and their slopes are close to each other. It appears to be a frequent situation that on MIPs the individual isotherms of similar compounds are straight lines with similar slopes in the $\log D-\log q$ plot [40]. It is not yet clear if this is a precondition for the isotherm overlap observed in Fig. 2. One should note, however, that some other well-known competitive isotherms behave the same way, i.e., the competitive isotherm coincides with the respective individual isotherms in the $\log D-\log q$ plot. One can easily show this property for the competitive Langmuir isotherm, which is widely used in chromatography [27] and in many technical problems, e.g., to describe the binding of surfactants by humic acids [41]. In any case, one should always see by measurements if the individual and the competitive $\log D-\log q$ plots overlap. This check requires only few extra measurements after the individual isotherms have been measured.

One should also note that at very high adsorbed concentrations the isotherms must go into saturation. The saturation range has not been investigated here but may be also important since many MIPs are imprinted with chiral templates (propranolol is one of them), and thus the adsorption of racemic mixtures on chiral MIPs may prove to be useful in preparative chiral separations [42].

\section{Conclusions}

An unsolved problem in the way of practical applications of MIPs has been that their isotherms in mixed solutions of the template and other compounds, i.e., in every practically relevant situation, have not been well researched. This paper has shown that in an apparently typical example equilibrium compositions in mixed solutions can be calculated by a simple graphical procedure. The precondition of this method is that the mixed solution (competitive) isotherms of the two compounds coincide with their respective individual isotherms in the $\log D$ $\log q$ isotherm plot. If this condition is satisfied then separations with MIPs can be quite simply predicted. Moreover, the four dimensional competitive isotherm can be easily obtained from the two individual isotherms and the selectivity of the MIP in mixed solutions can also be read from the individual isotherms.

\section{Acknowledgement}

The financial support of OTKA, Hungary (Grant No. K104724) is gratefully acknowledged. Z. Dorkó acknowledges the support of the Ernő Pungor Scholarship.

\section{References}

[1] MIPdatabase, patents in 2015. URL: http://mipdatabase.com/patents. php? year=2015

[2] Turiel, E., Martin-Esteban, A. "Molecularly imprinted polymers: Towards highly selective stationary phases in liquid chromatography and capillary electrophoresis." Analytical and Bioanalytical Chemistry. 378(8), pp. 1876-1886. 2004. https://doi.org/10.1007/s00216-003-2331-1

[3] Haginaka, J. "Monodispersed, molecularly imprinted polymers as affinity-based chromatography media." Journal of Chromatography B-Analytical Technologies in the Biomedical and Life Sciences. 866(1-2), pp. 3-13. 2008. https://doi.org/10.1016/j.jchromb.2007.07.019

[4] Pardeshi, S., Singh, S. K. "Precipitation polymerization: A versatile tool for preparing molecularly imprinted polymer beads for chromatography applications." Rsc Advances. 6(28), pp. 23525-23536. 2016. https://doi.org/10.1039/c6ra02784a

[5] Ansell, R. J. "Molecularly imprinted polymers for the enantioseparation of chiral drugs." Advanced Drug Delivery Reviews. 57(12), pp. 18091835. 2005. https://doi.org/10.1016/j.addr.2005.07.014

[6] Beltran, A., Borrull, F., Cormack, P. A. G., Marce, R. M. "Molecularlyimprinted polymers: Useful sorbents for selective extractions." TrACTrends in Analytical Chemistry. 29(11), pp. 1363-1375. 2010. https://doi.org/ 10.1016/j.trac.2010.07.020

[7] Martin-Esteban, A. "Molecularly-imprinted polymers as a versatile, highly selective tool in sample preparation." TrAC-Trends in Analytical Chemistry. 45, pp. 169-181. 2013. https://doi.org/10.1016/j.trac.2012.09.023

[8] Liu, Z. S., Zheng, C., Yan, C., Ga, R. Y. "Molecularly imprinted polymers as a tool for separation in CEC." Electrophoresis. 28(1-2), pp. 127 136. 2007. https://doi.org/10.1002/elps.200600544

[9] Moreno-Bondi, M. C., Navarro-Villoslada, F., Benito-Pena, E., Urraca, J. L. "Molecularly imprinted polymers as selective recognition elements in optical sensing." Current Analytical Chemistry. 4(4), pp. 316-340. 2008. https://doi.org/10.2174/157341108785914925

[10] Whitcombe, M. J., Chianella, I., Larcombe, L., Piletsky, S. A., Noble, J., Porter, R., Horgan, A. "The rational development of molecularly imprinted polymer-based sensors for protein detection." Chemical Society Reviews. 40(3), pp. 1547-1571. 2011. https://doi.org/10.1039/C0cs00049c 
[11] Li, S., Ge, Y., Piletsky, S. A., Lunec, J. (eds.) "Molecularly imprinted sensors: Overview and applications." First edition, Elsevier Science, Oxford, 2012.

[12] Lavignac, N., Allender, C. J., Brain, K. R. "Current status of molecularly imprinted polymers as alternatives to antibodies in sorbent assays." Analytica Chimica Acta. 510(2), pp. 139-145. 2004. https://doi.org/10.1016/j.aca.2003.12.066

[13] Zhang, J. L., Zhang, M. X., Tang, K. J., Verpoort, F., Sun, T. L. "Polymerbased stimuli-responsive recyclable catalytic systems for organic synthesis." Small. 10(1), pp. 32-46. 2014. https://doi.org/10.1002/smll.201300287

[14] Wulff, G., Liu, J. Q. "Design of biomimetic catalysts by molecular imprinting in synthetic polymers: The role of transition state stabilization." Accounts of Chemical Research. 45(2), pp. 239-247. 2012. https://doi.org/10.1021/ar200146m

[15] Yu, H., Yong, X. Y., Liang, J. Y., Deng, J. P., Wu, Y. P. "Materials established for enantioselective release of chiral compounds." Industrial \& Engineering Chemistry Research. 55(21), pp. 6037-6048. 2016. https://doi.org/10.1021/acs.iecr.6b01031

[16] Tashakori-Sabzevar, F., Mohajeri, S. A. "Development of ocular drug delivery systems using molecularly imprinted soft contact lenses." Drug Development and Industrial Pharmacy. 41(5), pp. 703-713. 2015. https://doi.org/10.3109/03639045.2014.948451

[17] Szekely, G., de Sousa, M. C. A., Gil, M., Ferreira, F. C., Heggie, W. "Genotoxic impurities in pharmaceutical manufacturing: Sources, regulations, and mitigation." Chemical Reviews. 115(16), pp. 8182-8229. 2015. https://doi.org/10.1021/cr300095f

[18] Huang, D. L., Wang, R. Z., Liu, Y. G., Zeng, G. M., Lai, C., Xu, P., Lu, B. A., Xu, J. J., Wang, C., Huang, C. "Application of molecularly imprinted polymers in wastewater treatment: A review." Environmental Science and Pollution Research. 22(2), pp. 963-977. 2015. https://doi.org/10.1007/s11356-014-3599-8

[19] Trotta, F., Biasizzo, M., Caldera, F. "Molecularly imprinted membranes." Membranes (Basel). 2(3), pp. 440-477. 2012. https://doi.org/10.3390/membranes2030440

[20] Verbic, T., Dorko, Z., Horvai, G. "Selectivity in analytical chemistry." Revue Roumaine de Chimie. 58(7-8), pp. 569-575. 2013. URL: http:// revroum.lew.ro/wp-content/uploads/2013/7/Art\%2001.pdf

[21] Dorko, Z., Verbic, T., Horvai, G. "Selectivity in analytical chemistry: Two interpretations for univariate methods." Talanta. 132, pp. 680-684. 2015. https://doi.org/10.1016/j.talanta.2014.10.018

[22] Dorko, Z., Verbic, T., Horvai, G. "Comparison of the single channel and multichannel (multivariate) concepts of selectivity in analytical chemistry." Talanta. 139, pp. 40-49. 2015. https://doi.org/10.1016/j.talanta.2015.02.030

[23] den Boef, G., Hulaniczki, A. "Recommendations for the usage of selective, selectivity and related terms in analytical chemistry." Pure and Applied Chemistry. 55, pp. 553-556. 1983. https://doi.org/10.1351/pac198855030553

[24] Vessman, J., Stefan, R. I., Van Staden, J. F., Danzer, K., Lindner, W., Burns, D. T., Fajgelj, A., Muller, H. "Selectivity in analytical chemistry (IUPAC recommendations 2001)." Pure and Applied Chemistry. 73(8), pp. 1381-1386. 2001. https://doi.org/10.1351/pac200173081381

[25] Thompson, M., Ellison, S. L. R., Wood, R. "Harmonized guidelines for single-laboratory validation of methods of analysis (IUPAC technical report)." Pure and Applied Chemistry. 74(5), pp. 835-855. 2002. https://doi.org/10.1351/pac200274050835

[26] Pap, T., Horvai, G. "Characterization of the selectivity of a phenytoin imprinted polymer." Journal of Chromatography A. 1034(1-2), pp. 99107. 2004. https://doi.org/10.1016/j.chroma.2004.01.064
[27] Guiochon, G., Felinger, A., Shirazi, D. G., Katti, A. M. "Fundamentals of preparative and nonlinear chromatography." Second edition, Academic Press, Boston, 2006.

[28] Carter, S. R., Rimmer, S. "Surface molecularly imprinted polymer coreshell particles." Advanced Functional Materials. 14(6), pp. 553-561. 2004. https://doi.org/10.1002/adfm.200305069

[29] Li, Z. W., Yang, G. L., Liu, S. B., Chen, Y. "Adsorption isotherms on nicotinamide-imprinted polymer stationary phase." Journal of Chromatographic Science. 43(7), pp. 362-366. 2005. https://doi.org/10.1093/chromsci/43.7.362

[30] Piperno, S., Bui, B. T. S., Haupt, K., Gheber, L. A. "Immobilization of molecularly imprinted polymer nanoparticles in electrospun poly(vinyl alcohol) nanofibers." Langmuir. 27(5), pp. 1547-1550. 2011. https://doi.org/10.1021/la1041234

[31] Yang, G., Wang, D., Li, Z., Zhou, S., Chen, Y. "Adsorption isotherms on aminoantipyrine imprinted polymer stationary phase." Chromatographia. 58(1-2), pp. 53-58. 2003. https://doi.org/10.1365/s10337-003-0012-Z

[32] Ren, Y. M., Yang, J., Ma, W. Q., Ma, J., Feng, J., Liu, X. L. "The selective binding character of a molecular imprinted particle for bisphenol a from water." Water Research. 50, pp. 90-100. 2014. https://doi.org/10.1016/j.watres.2013.11.042

[33] Andersson, L. I. "Application of molecular imprinting to the development of aqueous buffer and organic solvent based radioligand binding assays for (S)-propranolol." Analytical Chemistry. 68(1), pp. 111-117. 1996. https://doi.org/10.1021/Ac950668

[34] Ansell, R. J., Mosbach, K. "Magnetic molecularly imprinted polymer beads for drug radioligand binding assay." Analyst. 123(7), pp. 16111616. 1998. https://doi.org/10.1039/A801903g

[35] Bengtsson, H., Roos, U., Andersson, L. I. "Molecular imprint based radioassay for direct determination of $(S)$-propranolol in human plasma." Analytical Communications. 34(9), pp. 233-235. 1997. https://doi.org/10.1039/A703977h

[36] Haupt, K., Noworyta, K., Kutner, W. "Imprinted polymer-based enantioselective acoustic sensor using a quartz crystal microbalance." Analytical Communications. 36(11-12), pp. 391-393. 1999. https://doi.org/10.1039/A907844d

[37] Kamra, T., Xu, C., Montelius, L., Schnadt, J., Wijesundera, S. A., Yan, M., Ye, L. "Photoconjugation of molecularly imprinted polymer nanoparticles for surface-enhanced raman detection of propranolol." ACS Applied Materials \& Interfaces. 7(49), pp. 27479-27485. 2015. https://doi.org/10.1021/acsami.5b09500

[38] Kempe, H., Kempe, M. "Influence of salt ions on binding to molecularly imprinted polymers." Analytical and Bioanalytical Chemistry. 396(4), pp. 1599-1606. 2010. https://doi.org/10.1007/s00216-009-3329-0

[39] Yang, K. G., Berg, M. M., Zhao, C. S., Ye, L. "One-pot synthesis of hydrophilic molecularly imprinted nanoparticles." Macromolecules. 42(22), pp. 8739-8746. 2009. https://doi.org/10.1021/ma901761z

[40] Dorkó, Z., Tamás, B., Horvai, G. "Isotherm charts for material selection and method development with molecularly imprinted polymers and other sorbents." Talanta. 162, pp. 167-173. 2017. https://doi.org/10.1016/j.talanta.2016.10.027

[41] Rádi, J., Tóth, Z., Marton, A., Földényi, R. "Equilibrium studies of binding of cationic surfactants to sodium humate." Periodica Polytechnica Chemical Engineering. 58(Sup), pp. 27-33. 2014. https://doi.org/10.3311/PPch.7298

[42] Palovics, E., Szeleczky, Z., Bagi, P., Faigl, F., Fogassy, E. "Regularities between separations of enantiomeric and diastereoisomeric mixtures. Prediction of the efficiency of diastereomeric/enantiomeric separations on the basis of behaviour of enantiomeric mixtures." Periodica Polytechnica Chemical Engineering. 59(1), pp. 26-37. 2015. https://doi.org/10.3311/PPch.7328 